\title{
La colère dans la poésie épique, du Moyen Âge à la fin $\mathrm{du} \mathrm{XVI}{ }^{\mathrm{e}}$ siècle
}

Un envers de l'héroïsme?

\section{Bruno Méniel}

\section{OpenEdition Journals}

Édition électronique

URL : https://journals.openedition.org/crm/2002

DOI : 10.4000/crm.2002

ISSN : $1955-2424$

Éditeur

Honoré Champion

Édition imprimée

Date de publication : 15 avril 2004

Pagination : $37-48$

ISSN : 1272-9752

Référence électronique

Bruno Méniel, «La colère dans la poésie épique, du Moyen Âge à la fin du XVIe siècle », Cahiers de recherches médiévales [En ligne], 11 spécial | 2004, mis en ligne le 18 octobre 2007, consulté le 15 décembre 2022. URL : http://journals.openedition.org/crm/2002 ; DOI : https://doi.org/10.4000/crm. 2002

Ce document a été généré automatiquement le 15 décembre 2022.

Tous droits réservés 


\title{
La colère dans la poésie épique, du Moyen Âge à la fin du XVI ${ }^{\mathrm{e}}$ siècle
}

Un envers de l'héroïsme?

\author{
Bruno Méniel
}

1 Étudier la représentation de la colère qu'offre la poésie épique, c'est établir une relation entre un thème essentiel et un genre. La poésie épique se caractérise par un lexique, une gamme de métaphores traditionnelles, des motifs, des structures narratives, qui la conduisent à tenir sur la colère un certain discours. C'est ce discours que nous nous proposons de déchiffrer. En adoptant une perspective longue, nous tenterons de dégager une évolution, des constantes ou des changements, entre Moyen Âge et Renaissance.

2 Nous précisons que nous ne raisonnons pas en termes d'influence ni de transmission. Nous tentons plutôt de comprendre comment un genre littéraire évolue avec les mentalités collectives. Nous aborderons notre thème par le biais du lexique, ce qui nous permettra de dégager une pathologie de la colère; nous étendrons ensuite notre enquête aux comparaisons, dont nous déduirons une physique et une métaphysique de la colère; enfin nous nous attacherons aux structures, et nous proposerons une analyse politique de la colère.

Approche lexicale : pathologie de la colère

3 Tout personnage épique est un coléreux en puissance. Dans cette épopée archétypale qu'est La Chanson de Roland, tour à tour Ganelon, Marsile, Roland et Olivier s'abandonnent à la colère: Ganelon lorsqu'il est proposé par Roland comme ambassadeur auprès de Marsile, Marsile quand Ganelon profère devant lui les menaces de Charles, Roland lorsqu'il apprend qu'il sera placé à l'arrière garde, olivier, quand au cours de l'affrontement, le Sarrasin Falsaron s'exclame que la France perdra son honneur. On ne saurait écarter l'hypothèse d'un effort des jongleurs pour percer la psychologie du guerrier, mais il faudrait alors s'étonner de la rareté des analyses et du stéréotype des descriptions : l'épopée répugne à disséquer les sentiments et se contente d'en représenter les manifestations extérieures. On pourrait aussi expliquer la fréquente évocation de la colère par des raisons esthétiques : l'auditeur éprouverait du 
plaisir à voir le héros, perdant la maîtrise de lui-même et se débattant avec ses passions, descendre de son piédestal ; à observer d'en haut celui qu'il admirait d'en bas. Il faudrait alors que l'on s'attardât sur les scènes de colère, qu'elles fussent développées et traitées pour elles-mêmes. C'est rarement le cas : d'ordinaire, la colère est évoquée en passant. Toujours, néanmoins, à des endroits cruciaux du récit. Il nous semble donc qu'il convient en premier lieu de s'interroger sur sa fonction.

4 La colère est, dans la chanson de geste, un motif investi d'une fonction inaugurale et d'une fonction structurelle. La colère est à l'origine de l'action épique dans de nombreux poèmes : c'est vrai pour l'Iliade, et c'est vrai pour les chansons des barons révoltés : Girart de Roussillon ou Renaut de Montauban naissent à leur destin par la colère. Si l'on envisage dans son ensemble l'histoire racontée, la colère joue un rôle moteur. Si l'on considère, en revanche, les épisodes, elle marque souvent un revirement de l'action. Lorsqu'il se met en colère, un personnage avance au premier plan; il devient le protagoniste, c'est-à-dire à la fois « le soldat qui combat au premier rang » et "l'acteur qui occupe le premier rôle ». Le personnage, passif parce qu'il observait ou même subissait la violence, devient actif dès lors qu'il accueille celle-ci et la concentre en lui.

5 Avant tout, les tournures qui, dans la chanson de geste, expriment la colère prennent valeur de signe : elles visent à ponctuer l'action, à signaler un revirement, à dramatiser un épisode ${ }^{1}$; mais ce serait paresse de ne voir en elles que des formules stéréotypées, vidées peu à peu de leur signification littérale, des hyperboles qui ne seraient plus perçues comme telles. Il est préférable de se rappeler qu'elles résultent de choix innombrables opérés par une longue tradition. Il convient donc d'être attentif au discours implicite qui, par un lent travail de sédimentation, s'est fixé en elles². Il y a d'abord le «maltalent»: une mauvaise intention, une disposition hostile. Dans Le Charroi de Nîmes, le maltalent semble une colère à laquelle le héros serait porté par propension atavique. En effet, lorsque Louis, dans deux laisses parallèles, reproche à Guillaume d'en être plein, le héros répond une fois "si furent mi parent $»^{3}$ et une autre "s'orent mi ancesor " ${ }^{4}$ (ainsi furent mes ancêtres). Les mots "ire " et "corrouz " désignent à la fois la souffrance morale, le chagrin et la colère. Ils peuvent renvoyer à une blessure d'amour propre. En tout cas, la colère est liée à la souffrance, comme l'indique l'association de synonymes «dolant et correços ». Le mot "rage » reste tributaire de son origine médicale. Charlemagne dit à Ganelon « El cors vos est entree mortel rage $»^{6}$. Lorsque la colère ne vient pas du corps, elle passe par lui. Nous voyons ainsi se dégager un premier type de colère épique. Cette colère triste, morbide, délétère serait perçue comme une force agressive dont le sujet serait heurté, avant que, découvrant qu'elle peut le détruire, il ne l'oriente vers autrui.

6 Ailleurs, le chevalier «ad tel doel pur poi d'ire ne fent/A ben petit que il ne pert le sens $»^{7}$, " a pou d'ire n'enraige $»^{8}$, "le sens cuide changier $»^{9}$ ou "le sens cuide desver $»^{10}$ ou "le sens quide marir $»^{11}$ : il croit perdre la raison, il est littéralement hors de lui, il ne se contrôle plus. La colère épique du second type relève donc de la folie et de la démesure. Elle caractérise celui qui est sorti du bon sens, le desreé. Elle tire son origine du scandale de la déraison; au lieu d'éliminer l'irrationnel, le sujet se laisse submerger par lui. Au style formulaire et à l'accumulation des épisodes, caractéristiques de la chanson de geste, correspond la psychologie du colérique, voué à la répétition : le héros épique des XII ${ }^{\mathrm{e}}$-XIII ${ }^{\mathrm{e}}$ siècles doit faire se succéder les massacres 
et les exploits pour assurer sa survie. L'univers de la chanson de geste est celui de la rechute, de l'itération.

Dans la poésie épique de la Renaissance, le lexique de la colère se réduit. Par exemple, le mot «maltalent ", qui se rencontre encore à la fin XVI ${ }^{\mathrm{e}}$ siècle, chez des prosateurs comme Montaigne, Brantôme, Pasquier, est quasiment absent de la poésie épique.

Le mot « cholère ", qui désignait au Moyen Âge une humeur, la bile jaune, ne prend son sens moderne d'« état affectif violent " qu'au XVe siècle. Le Französisches Etymologisches Wörterbuch, renvoyant aux notes lexicologiques d'A. Delboulle, attribue au Livre Caumont (1416) la première occurrence du mot dans son acception actuelle ${ }^{12}$. Le mot fonctionne comme doublet de « ire » et de " courroux " jusqu'au XVII ${ }^{\mathrm{e}}$ siècle. En même temps que la graphie "cholère " cède du terrain, le vocable se spécialise dans un registre courant et dans le registre technique de la médecine, alors que les termes « ire » et "courroux» relèvent de plus en plus du registre élevé. Pendant toute la Renaissance, la colère est conçue en fonction du système des humeurs, puisque cette passion correspond à un tempérament particulier :

Qui jecoris cordisque calido et sicco temperamento sunt bilis flavae copiam vel ex optimis et bene temperatis alimentis exaggerant [...]. In iracundiam, in audaciam et vindictam propensio : somnus levis nec profundus, magna corporis jactatione inquietus : insomnia belli, furoris, iracundiae splendorisque plena $[. . .]^{13}$.

[...] nihil jam ambigi debet [...] eam [appetitionem] [...] quae ad iram proclivis est et thumôsis dicitur, in corde, quod unum inter omnia viscera maximo ardet incendio, ut hos possit impetus inflammare $[. . .]^{14}$.

Les poètes de la Renaissance partagent cette représentation médicale de la colère. Celle-ci apparaît dans l'épopée sous l'aspect de la fureur, qui est naturellement associée à la chaleur. Les mots employés dans La Savoysiade d'Honoré d'Urfé pour évoquer celle qui anime le More Argorant, après qu'il a été blessé, appartiennent au champ sémantique du feu :
Argorant, que l'offence à la vangeance appelle,
Voyant le long du bras que le sang luy ruisselle,
Transporté de fureur qui le brusle au dedans,
Les yeux comme flambeaux en son courroux ardantz,
Sur le jeune Gaston, pour l'accabler, s'eslance $[. . .]^{15}$.

Si la colère et la rage sont d'ordinaire jugées avec sévérité, la fureur guerrière apparaît comme une passion beaucoup plus noble. Faisant implicitement référence au Phèdre de Platon, Montaigne écrit que les maitres de la philosophie attribuent «les causes des eslancements extraordinaires de nostre esprit [...] à un ravissement divin, à l'amour, à l'aspreté guerriere, à la poësie, au vin ${ }^{16}:$ il glisse parmi les quatre délires platoniciens la fureur guerrière. La fureur du combattant est donc un transport divin. Ainsi, dans $L a$ Savoysiade, la fureur du More Selidan peut être comparée à un fléau de Dieu :

Luy, avec Almançor ravageant toutte Espagne,

Avoit de sang chrestien arrosé la campagne

Lors que, plain de fureur aynsi qu'un fleau de Dieu,

Le feu, le sang, la mort ilz portoient en tout lieu [... ${ }^{17}$.

11 Cependant, avec la bonne impulsion qu'est la fureur s'allie parfois la mauvaise qu'est la rage. Cet effacement des différences entre les passions se produit chez Feragut, le principal guerrier more de L'Espagne conquise :

Une ardente fureur rampe dedans ses os

Il chancelle de rage, et poulse de furie ${ }^{18}$. 
Provoqué en combat singulier par le protagoniste, Ferry, Feragut réagit étrangement :

Le paian à ces motz horriblement enrage,

Et de son propre fer furieux il s'outrage.

Se bat le chef du poing il faict bruire ses dents,

Et jecte sur Ferry mille regards ardents :

Puis luy dict en fureur : $O$ vile creature

Qui de ta triste vie as si petite cure !

Que n'as tu mille corps, afin que mille coups

Les fissent tous mourir en mon ardent courroux?

Trop petit est ton sang pour saouler ma vengeance,

Et trop peu je verray ta folle outrecuidance

Punie par ta mort : mais approche insensé,

Viens recevoir la mort pour m'avoir offensé $[. . .]^{19}$.

Ici s'installe un climat de démence. Alors même que Feragut accuse son adversaire de «folle outrecuidance », il se comporte en fou. Offensé, il tourne la colère vers lui-même avant de la diriger contre son adversaire : les différences s'estompent entre la rage et la fureur, entre soi et l'autre. La colère est une force qui appelle la mort : si l'on ne veut pas que ce soit la sienne, il faut que ce soit celle de l'autre.

L'intérêt pour la colère qui se manifeste dans le dernier tiers du XVI ${ }^{\mathrm{e}}$ siècle est sans doute lié à l'interrogation sur les causes profondes des guerres de religion, qui peuvent être considérées tantôt comme un mouvement irrationnel d'autodestruction, tantôt comme une purification voulue par Dieu. L'approfondissement de l'analyse psychologique de la colère aboutit à révéler l'ambivalence de la pulsion. Parallèlement se tisse autour d'elle un réseau d'analogies qui peut permettre d'accéder aux raisons secrètes de cette ambivalence.

Approche métaphorique : physique et métaphysique de la colère

Comme l'écrit Dominique Boutet, «la chanson de geste donne à voir sans vraiment décrire : son moyen privilégié est l'esquisse stylisée $»^{20}$. Il arrive cependant que le motif de la colère soit quelque peu développé, comme dans ce passage de Jehan de Lanson :

Quant duc Jehan l'entent, le cuer ot moult yré ;

Ung mot ne pot parler, tant ot le cuer enflé ;

De grant yre et de duel ot le vis alumé

Car onquez en sa vie ne fu si tourmentée ${ }^{11}$.

L'hyperbole dit l'excès qui est le propre de la colère; dans le poème épique de la Renaissance, comme déjà chez Homère et Virgile, cette démesure s'exprime au moyen de comparaisons, qui ont un effet d'amplification. Comparée aux forces de la nature, la colère s'inscrit dans l'ordre cosmique. Elle s'abat sur les hommes avec la même fatalité inéluctable que la pluie, la grêle ou la peste. Par exemple, Nicolas de Montreux rapproche la fureur de la puissance qui anime les torrents :

Nul ne peult la fureur inhumaine arrester,

Non plus que d'un torrent que $1[$ ']on voit se jeter

Du hault d'un mont pointu, et qui superbe roulle

D'un roulement plus vif qu'une legere boulle ${ }^{22}$.

Plus loin, il compare l'affrontement de deux guerriers à celui de deux vagues avançant l'une vers l'autre :

Ainsi que sur la mer on voit deux onde fieres

Dont le sein est enflé de deux ventz adversaires

Venir se rencontrer bruiantz d'aspre fureur

Qui font aux navigantz palle esprouver la peur. 
Puis au choc inhumain qui les bateaux enfondre

En sables areneux l'une et l'autre se fondre :

Ainsi courent ces preux de mille preux vainqueurs [... ${ }^{23}$.

18 Ailleurs encore, une comparaison homérique assimile la colère du païen Armende à celle d'un serpent :

Ainsi qu'un roux aspic, que le passant lassé
En passant son chemin à du baston blessé,
Se dresse contre luy, et d'une rage ardante
Allonge le chef torts, et la langue siflante,
S'eslance contre luy, tasche de le choquer
Et le chocquant, son froc cruellement picquer
Il cherche le trespas ou la juste vangeance
De l'injure receüe et du coup qui l'offence,
Car cest ardeur bouillonne à soufflementz esgaux
Es coeurs des animez, et des fiers animaux.
Tout ce qui vit en terre esmeu de ceste envie
Veut par la mort d'autruy, rendre vive sa vie [...] $]^{24}$.

La fureur est assimilée à ces puissances naturelles qui fascinent et terrifient l'être humain. Le désir de tuer est trop mystérieux et trop terrifiant pour émaner de l'homme. Les comparaisons expriment que la fureur guerrière vient d'ailleurs, qu'elle est inéluctable et incontrôlable. Nous comprenons dès lors la nature de la limite qui est franchie lors du passage à la violence : c'est la frontière entre le profane et le sacré.

Comme l'a montré Roger Caillois, le sacré est par essence ambigu : en lui se conjuguent le pur et l'impur, la sainteté et la souillure. Au temps des troubles civils, la colère est pour les uns la puissance qui anime le guerrier de Dieu, bras armé de la Providence ; pour d'autres, elle est un péché d'autant plus grave qu'il est accompli au nom de la religion. Ainsi, certains poètes épiques affirment que la religion n'est qu'un prétexte, que l'on ne saurait tuer au nom de Celui qui demande d'aimer son prochain. L'Erynne françoyse d'Hubert-Philippe de Villiers dit des guerres qui déchirent la France que

ces divisions

Sont soubz pretexte sainct de deux religions :

Dont le mesme subject par diverse doctrine,

Diversement mené cause nostre ruine,

Et des noms factieux la dure nouveauté

Amorce la fureur d'un et d'autre costé $[. . .]^{25}$.

21

\section{En effet, la violence ne peut avoir pour cause véritable la religion.}

À preuve que la colère est un péché, les signes se multiplient d'une punition divine. Par exemple, dans l'œuvre d'un poète anonyme, L'argument de la victoire que le Roy s'est acquise sur les Ristres (1588), il est dit que les reîtres qui déferlent d'Allemagne sont un châtiment envoyé par Dieu à la France coupable de s'être abandonnée à la colère :

Car du Septentrion a jadis la Judee

Sentit l'ire de Dieu sur sa teste guidee,

Et du Septentrion sent la France en effet

Combien l'ireus peché au Souverain desplait ;

Lequel a suscité le Ristre en ses contrees

Et luy a concedé faciles les entrees $[. . .]^{26}$.

En général, dans la Bible, les malheurs d'Israël viennent du nord (Jr 4, 6; 6, $1 ; 15,12$; $50,3 ; \mathrm{Jl} 2,20)$. Le texte que nous citons fait sans doute allusion à l'invasion de Nabuchodonosor. 
Cette perpétuation de la colère s'exprime par la structure de l'action: au cours d'un combat collectif, un personnage en tue un autre ; un troisième assiste à la scène, s'irrite de voir son compagnon occis et se jette sur l'agresseur. La Henriade de Sébastien Garnier perfectionne ce dispositif. Le récit de la bataille d'Ivry y obéit à un schéma qui enchaîne combat individuel et combat collectif: dans la mêlée, un guerrier français tue un guerrier espagnol ; pour venger celui-ci, les Espagnols s'acharnent sur le Français, que vient secourir un compagnon. Le deuxième Français tue un deuxième Espagnol, et ainsi de suite. Le désir de vengeance et l'entraide sont les deux ressorts de l'action. Dans L'Espagne conquise de Nicolas de Montreux, le païen Armende, est mis en rage par la mort de son cousin, qui vient d'être tué par Regnault :

Armende le felon, l'audatieux, le fort,

Qui cruel avoit mid mille françois à mort,

Le sang de son cousin faict que le sien buollongne,

De vageance $[$ sic $]$ asseüvee, et de rage fris $[s]$ onne $[. . .]^{33}$. 
violence se diffuse de proche en proche : par un phénomène de contamination, le sang du défunt met en effervescence celui du survivant; le sang qui noircit et se fige communique son énergie au sang rouge et fluide. La difficulté vient de ce que seule la violence permettrait d'éteindre la violence, comme le dit Alexandre de Pontaymeri :

La cruauté finit par un semblable à soy,

Elle mesme se juge et se donne la loy:

Le seul contrepoison d'un effort tyrannique

Est d'estancher le sang par le sang de l'inique ${ }^{34}$.

31 Face à la colère, toujours encline à se propager, doit se dresser l'autorité du souverain. Fondamentalement, la chanson de geste exprime une idéologie vassalique favorable au renforcement du pouvoir royal : elle dénonce les dangers de la faide, cette vengeance privée qui place le roi sur un pied d'égalité avec ses vassaux et qui fait de la guerre un moyen d'obtenir justice. Pourtant le genre évolue au cours du XII siècle et aboutit à ce que Dominique Boutet présente comme « une crise de la représentation de la royauté »35. La faiblesse et l'ingratitude du roi provoquent la colère du vassal. La pulsion de violence trouve sa source dans le sentiment d'être victime d'une injustice et notamment de voir son honneur dénié. Ainsi, dans Aliscans, qui date des années 1180, Guillaume réagit avec emportement à l'accueil froid que lui fait à Laon le roi Louis :

Diex, dist li quens, com or puis esragier

Quant ciex se paine de moi contaloier

Ki me déust aloser et prisier,

Desor tous hommes amer et tenir chier ${ }^{36}$ !

C'est bien la représentation de la royauté qui est en cause, car, à cette époque, le pouvoir royal est bien assis. Dominique Boutet souligne qu'au cours du XII ${ }^{e}$ siècle, la représentation du roi et la réalité historique sont en décalage l'une par rapport à l'autre :

Par un effet de " chassé-croisé 》, le début de ce siècle, qui est une époque de morcellement féodal, de fragmentation de l'espace politique, est le temps d'une conscience pleine, qui se reconnaît dans un monde ordonné, dans une figure royale littéraire ferme et unitaire; la fin du XII siècle et le début du suivant, qui sont marqués par une centralisation croissante, par une unification politique de plus en plus sensible et par un accroissement considérable du pouvoir royal, sont l'époque de la fragmentation dans l'ordre des représentations mythiques, avec une figure royale fuyante, car éclatée ${ }^{37}$.

33 À la fin $\mathrm{du} \mathrm{XVI}{ }^{\mathrm{e}}$ siècle, coexistent les deux images de la royauté, suivant les positions politico-religieuses des poètes. Les "politiques", qui s'illustrent dans le poème héroïque, présentent un roi fort, juste, reconnaissant, et donc obéi et respecté, parce qu'il apaise la colère en interrompant le cycle de la vengeance par un châtiment exercé au nom de la collectivité. Les poètes ligueurs ou les protestants zélés, qui pratiquent le poème romanesque ou le poème de combat, évoquent un monde où la justice d'État a du mal à s'imposer, qu'elle soit faible ou corrompue. La Guisiade, poème composé par un ligueur anonyme, vitupère l'injustice et l'ingratitude d'Henri III, qui a trahi de fidèles alliés en ordonnant le double meurtre de Blois. Le manque de reconnaissance est aussi au cœur de La Lydiade de François Descallis : alors que le héros, Alcestes, sauve par ses exploits militaires le roi de Lydie, celui-ci lui refuse la main de sa fille. Dans «Princes", d'Aubigné dénonce l'ingratitude d'Henri IV envers les gentilshommes qui l'ont loyalement servi et ses complaisances pour les ligueurs repentis :

Ce thresor precieux de nostre liberté

Nous est par les ingrats injustement osté : 
Les ingrats, insolents à qui leur est fidelle,

Et liberaux de crainte à qui leur est rebelle [... $]^{38}$.

Le régime de la faveur empêche une juste rétribution des services rendus, de sorte qu'enfle le mécontentement et la colère.

En faisant une telle place à la colère, l'épopée exprime combien sont mal vécues les relations d'interdépendance entre le souverain et les nobles; elle ne peut que louer, chez l'un et chez les autres, la maîtrise de soi. Pour les hommes du Moyen Âge, la colère est en effet une dangereuse altération. Le verbe "corocier» vient du latin "corrumpere"; il est attesté à la fois dans le sens physique d'«endommager, maltraiter » et dans le sens moral d' «irriter vivement ». En effet, le courroux est une altération de l'âme ; quelque chose de l'humanité s'y gâte irrémédiablement. Dans la chanson de geste, la colère manifeste la puissance, mais elle trahit aussi l'orgueil. C'est pourquoi elle attribue à ses héros les plus nobles la faculté de pardonner. Il en va ainsi de Guillaume, qui déclare :

Deus ne fist ome qui tant m'ait corocié,

Se tant puet faire que il viegne à mon pié,

Ne li pardoinse de gré et volentiers ${ }^{39}$.

Selon Micheline de Combarieu, «Ce type de pensée et de conduite est [...] présenté comme un idéal plus que comme une norme. $»^{40}$

Dans la poésie épique des guerres de religion, l'aptitude au pardon, et plus généralement à la clémence, est attribuée en particulier au personnage d'Henri IV, érigé en héros néo-stoïcien par ceux qui défendent l'idéologie des " politiques ». Cette clémence procède à la fois de la maîtrise sénéquienne des passions et de la charité chrétienne. Dans l'œuvre de Sébastien Garnier, Henri IV sait qu'une fois qu'on a laissé éclater la colère de la soldatesque, il est difficile de la contenir. Soucieux d'épargner le sang de ses sujets, il empêche ses troupes d'abuser de leur force :

Or le Roy redoutant le soldart animé

Qu'il ne peust une fois en son ire enflammé,

L'empescher de tuer : tenant l'espee nue,

Crioit tant qu'il pouvoit gallopant par la ruë,

Prenez compassion, cessez l'inimitié

Que vous avez contr'eux, les prenans à pitié,

Regardez, mes amis, je vous pri' qu'ils sont hommes,

François de Nation aussi bien que nous sommes [... ${ }^{41}$.

Garnier ne réserve pas au Roi la faculté de contrôler ses passions; Châtillon se réconcilie avec La Noue en déclarant : «La colere souvent l'homme sage maistrise » ${ }^{42}$. Sébastien Garnier conforme donc l'attitude du Roi et de ses capitaines à un idéal néostoïcien de maîtrise des passions.

39 Alexandre de Pontaymeri, s'adressant à Henri IV, lui déclare : « la pitié/Maistresse de ton cœur, chasse l'inimitié,/Loing de tes passions $»^{43}$. Selon un lieu commun, seul peut commander aux autres celui qui commande en premier lieu à lui-même : à la bataille de Coutras, Henri manifeste cette qualité :

Car l'homme valeureux, de sa gloire jaloux,

Ayant tout surmonté doit vaincre son couroux,

Pardonner aux vaincus, et d'un tiède courage

Alentir la fureur du devorant carnage ${ }^{44}$.

À la fin de l'ouvrage, l'attitude de Philippe II contraste singulièrement avec celle d'Henri IV. Apprenant qu'à la bataille de Villemur, le comte de Ladron, général des 
Lorrains, a abandonné le combat, et qu'Antoine-Scipion de Joyeuse, chef des ligueurs, a été tué, le monarque espagnol entre en une telle colère qu'il fait une crise de " haut mal », de sorte qu'« Il pert tout son maintien, et sa grandeur Royale » ${ }^{45}$. Ainsi, l'épopée oppose le bon monarque, qui domine sa colère et apaise celle des autres, et le mauvais, qui se laisse envahir et détruire par elle.

41 La façon dont le poème épique du Moyen Âge et celui de la Renaissance présentent la colère fait apparaître entre eux des ruptures et des continuités. Nous passons d'une poésie dominée par la stylisation et le stéréotype, où les formules mettent en évidence la perpétuation de la violence, à une poésie fondée sur une esthétique de la varietas, qui multiplie les comparaisons, soulignant les particularités physiologiques de la colère et assimilant celle-ci à un phénomène naturel. Considérée sur une longue période, cependant, la relation de l'épopée au politique n'est pas dépourvue de permanence : les rapports difficiles entre le roi et les grands seigneurs offrent au poète un sujet privilégié. Le poème épique condamne la colère comme une passion nocive, qui témoigne de l'orgueil, de la démesure, et confine à la folie. Si l'on en croit les chansons de geste et, au XVI ${ }^{\mathrm{e}}$ siècle, les œuvres de poètes " politiques » comme Sébastien Garnier ou Alexandre de Pontaymeri, l'héroïsme du courage doit céder le pas à celui de la sagesse et la communauté se ressouder autour du monarque.

\section{NOTES}

1.Voir Dominique Boutet, Jehan de Lanson, Technique et esthétique de la chanson de geste au XIII siècle, Paris, PENS, 1988, p. 100.

2.Nous sommes ici redevable aux analyses de Georges Kleiber, Le Mot « ire » en ancien français : $\mathrm{XI}^{e}-\mathrm{XIII}{ }^{e}$ siècles : essai d'analyse sémantique, Klincksieck (Bibliothèque française et romane ; Série A : Manuels et études linguistiques, 41), 1978, 488 p.

3.Le Charroi de Nîmes, v. 296.

4.Ibid., v. 302.

5.Prise d'Orange, v. 1024.

6.Chanson de Roland, v. 747 : « Au corps vous est entrée mortel rage »

7.Ibid, v. 303-305: « [Ganelon] éprouve une telle douleur qu'il pense éclater de colère ; peu s'en faut qu'il ne perde le sens.»

8.Orange, v. 1054.

9.Le Couronnement de Louis, v. 1112.

10.Ibid., v. 2658.

11.Aliscans, v. 43.

12.Voir A. Delboulle, « Notes philologiques » in Revue d'Histoire Littéraire de la France, vol. 6 (1899), p. 460 ; Le Livre Caumont, où sont contenus les dits et enseignemens du seigneur de Caumont composés pour ses enfans, l'an 1416, éd. J.-E. Galy, Paris, J. Téchener, 1845, p. 50. 13.Jean Fernel, Pathologia, II, XV, in Universa medicina, avec les notes de Johannes et Othon Heurnus, Trajecti ad Rhenum, Gisberti a Zyll et Theod. Ab Ackersdijck, 1656, p. 235, col. 1 - p. 236, col. 2 : « Ceux qui ont le foye et le cœur d'un tempérament chaud et sec, amassent beaucoup de bile jaune, mesme des alimens plus sains et bien temperez 
[...] ; l'on est enclin à la cholere, à l'audace et à la vengeance ; le sommeil est leger, non profond, plein d'inquiétude ; les songes ne sont que de fureur, de guerre, de cholere, et de splendeur [...] » (Pathologie, trad. A.D.M., Paris, Vve de Jean Le Bouc, 1646,

pp. 136-137).

14.Id., Physiologia, V, XIX, ibid., p. 126, col. 2, A : « on ne doit point du tout douter que [...] l'appetit de colere, et qui est appellé par les grecs Tumosis c'est à dire colère, est et reside dedans le cœur, qui seul d'entre tous les visceres brusle d'une tres-grande chaleur, afin qu'il puisse enflammer et allumer ces impetuosités [...] » (Les sept livres de la Physiologie [...], traduits en françois par Charles de Saint-Germain, [...], Paris, Jean Guignard le jeune, 1655 ; réimp. Paris, Fayard (Corpus des CEuvres de Philosophie de langue française), 2001, p. 440).

15.Honoré d'Urfé, La Savoysiade, manuscrit. Ars 2959, II, v. 552-556.

16.Montaigne, Essais, III, 5, éd. P. Villey, PUF, 1966, p. 844 C.

17.Ibid., III, v. 201-204.

18. [Nicolas de Montreux], L'Espagne conquise, $1^{\mathrm{e}}$ partie, 1597, XII, p. 197, v. 6-7 ; poulse : palpite.

19.Ibid., XII, p. 196, v. 6-17.

20.Dominique Boutet, op. cit., p. 113.

21.Jehan de Lanson, v. 505-508, cité ibid., p. 142.

22.[Nicolas de Montreux], L'Espagne conquise, $1^{\mathrm{e}}$ partie, 1597, IX, p. 128, v. 22-25. Cf. Il., IV, v. 452-455.

23.Ibid., $2^{\mathrm{e}}$ partie, 1598, XVII, p. 297, v. 17-23.

24.Ibid., I, p. 13, v. 13 - p. 14, v. 4. Cf. Il., III, v. 33-35 ; Én., II, v. 379-382.

25.Hubert-Philippe de Villiers, Cinq livres de l'Erynne françoise, Paris, Jean Le Blanc, 1585, II, f. Ciiij v ${ }^{\circ}$, v. 7-12.

26.L'argument de la victoire que le Roy s'est acquise sur les Ristres (1588), p. 5, v. 18-23. Voir Jr 25, 8-9: «Eh bien! ainsi parle le Seigneur le tout puissant : Puisque vous n'écoutez pas mes paroles, je donne ordre de mobiliser tous les peuples du nord - oracle du Seigneur -, en faisant appel à Nabuchodonosor, roi de Babylone, mon serviteur, et je les amène contre ce pays, contre ses habitants - et contre toute ces nations voisines [...] ». 27.Jean-Charles Payen, « Les épopées de la démesure et de la révolte » [extrait du Motif du repentir dans la littérature française médiévale, Genève, Droz, 1967], Denis Hüe, dir., L'orgueil a desmesure. Études sur Raoul de Cambrai, Orléans, Paradigme, 1999, p. 228.

28.Cf. Mc 1. 23-26.

29.Agrippa d'Aubigné, Les Tragiques, éd. Jean-Raymond Fanlo, Paris, H. Champion (Textes de la Renaissance, 6), 1995, VI, v. 953-954, t. I, p. 628.

30.Voir Émile Durkheim, Les Formes élémentaires de la vie religieuse, Paris, PUF, 1960. 31.Prise d'Orange, v. 872-873, trad. D. Boutet in Le Cycle de Guillaume d'Orange, Librairie Générale Française, 1996, p. 227 :

Arragon, voyant cela, devient presque enragé:/La douleur et la colère le rendent presque fou.

32.[Nicolas de Montreux], L'Espagne conquise, $1^{\mathrm{e}}$ partie, 1597, XI, p. 168, v. 18-19.

33.Ibid., $2^{\mathrm{e}}$ partie, Paris, Abraham Saugrain, 1598, I, p. 13, v. 13-16.

34.Alexandre de Pontaymeri, La Cité de Montélimar, (s.l.), 1591, I, p. 24, v. 18-21.

35.Dominique Boutet, Charlemagne et Arthur ou le roi imaginaire, Paris, Champion, 1992, p. 609 .

36.Aliscans, v. 2479-2482, trad. D. Boutet in Le Cycle de Guillaume d'Orange, éd. cit., p. 367 : Dieu, dit le comte, comme la colère m'étouffe,/Quand je vois s'appliquer à me couvrir 
de sarcasmes/Celui qui aurait dû me célébrer et m'honorer,/M'aimer et me chérir plus que tout homme!

37.Dominique Boutet, op. cit., pp. 610-611.

38.Agrippa d'Aubigné, op. cit., II, v. 627-630, éd. cit., t. I, p. 202.

39.Le Couronnement de Louis, v. 1735-1737, trad. p. 129:

Dieu n'a pas créé d'homme qui m'ait à ce point courroucé/Que, s'il en vient à me baiser le pied,/Je ne lui pardonne de bon cœur.

40. Micheline de Combarieu du Grès, «La Violence dans Le Couronnement de Louis « in Mélanges de litterature du moyen age au XXe siècle, offerts à Mademoiselle Jeanne Lods [...],

Paris, École Normale Supérieure de Jeunes Filles, 1978, p. 144.

41. Sébastien Garnier, Les Huict Premiers Livres de la Henriade, Blois, Veuve B. Gomet, 1594, I, p. 20, v. 23-30.

42. Ibid., I, p. 13, v. 17.

43. Pontaymeri, Le Roy triomphant, Cambrai, p. 10, v. 17-9.

44. Ibid., p. 13, v. 19-22.

45. Ibid., p. 140, v. 22. Selon ses biographes, Philippe II souffrait de la goutte et de fièvre. L'épilepsie ne semble pas avoir fait partie de ses maladies.

\section{AUTEUR}

BRUNO MÉNIEL

Université Rennes-II 